\title{
Multidisciplinary management of tracheobronchial injury
}

\author{
Jacques Boutros $\mathbb{1}^{1}$, Charles-Hugo Marquette $\mathbb{( i l}^{1,2}$, Carole Ichai ${ }^{3}$, Sylvie Leroy ${ }^{1,4}$ and \\ Jonathan Benzaquen ${ }^{1,2}$
}

${ }^{1}$ Université Côte d'Azur, Centre Hospitalier Universitaire de Nice, Dept of Pulmonary Medicine and Oncology, Nice, France. ${ }^{2}$ Université Côte d'Azur, CNRS UMR7284, Inserm U1081, Institute of Research on Cancer and Ageing (IRCAN), Nice, France. ${ }^{3}$ Université Côte d'Azur, Centre Hospitalier Universitaire de Nice, Dept of anesthesia and critical care, Nice, France. ${ }^{4}$ Université Côte d'Azur, CNRS UMR 7275 Institut de Pharmacologie Moléculaire et Cellulaire, Sophia Antipolis, France.

Corresponding author: Jacques Boutros (boutros.j@chu-nice.fr)

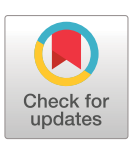

Copyright

This version is distributed under the terms of the Creative Commons Attribution Non-Commercial Licence 4.0. For commercial reproduction rights and permissions contact permissions@ersnet.org

Received: 26 May 2021 Accepted: 16 Sept 2021

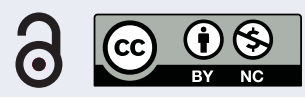

Shareable abstract (@ERSpublications)

Tracheobronchial injuries are heterogeneous, comprising rare and potentially life-threatening scenarios. Post-intubation tracheal/post-traumatic tracheobronchial injuries need multidisciplinary evaluation and some cases may be managed conservatively. https://bit.ly/3kug4ud

Cite this article as: Boutros J, Marquette $\mathrm{C}-\mathrm{H}$, Ichai $\mathrm{C}$, et al. Multidisciplinary management of tracheobronchial injury. Eur Respir Rev 2022; 31: 210126 [DOI: 10.1183/16000617.0126-2021].

\section{Abstract}

Tracheobronchial injury is a heterogeneous entity comprising multiple rare and potentially life-threatening scenarios. We performed a systematic literature review focusing on post-intubation tracheal injuries (PiTIs) and post-traumatic tracheobronchial injuries (PTTBIs).

PiTIs are often longitudinal lacerations of the middle third of the membranous trachea. Subcutaneous emphysema of the face and trunk following tracheal intubation should immediately trigger the diagnosis. Diagnosis may be suspected on the chest computed tomography (CT) and should be confirmed by bronchoscopic examination. Conservative management is encouraged for a spontaneously breathing or stable patient on noninvasive ventilation. Surgical repair is mandatory when mechanical ventilation is required and if bridging of the injury is impossible.

PTTBIs are often associated with other severe injuries. Patients often present with massive subcutaneous emphysema and intractable pneumothorax. Diagnosis may be suspected on the chest CT and should be confirmed by bronchoscopic examination. Early surgical repair is indicated. In selected patients, conservative management can be considered.

\section{Introduction}

In daily practice, anaesthesiologists and intensivists have limited experience in the field of tracheobronchial injuries due to the lack of publications in the literature. This is a teaching document providing an extensive review of the scientific literature discussing risk factors, manifestations and management of tracheobronchial injuries. We chose to separate post-intubation tracheal injuries (PiTIs) from post-traumatic tracheobronchial injuries (PTTBIs) in our report since these two groups of injuries are inherently different in their mechanisms, endoscopic aspects, clinical manifestations, prognosis and management. We chose not to discuss the specific cases of tracheobronchial injuries occurring after cervico-thoracic surgery or minimally invasive endoscopic techniques such as rigid bronchoscopy and endoscopic ultrasonography.

We performed a systematic literature review of the scientific literature in PubMed for articles in English describing case series of tracheal laceration or injury in adults. The period chosen for the study was from January 1990 to January 2021. For the PiTI review, the search was defined using the terms "trachea", "tracheal”, "bronchus”, "bronchial”, "rupture”, "laceration”, "injury” "intubation” and "postintubation” in the title of the article. For the PTTBI review, we searched for the terms "trachea", "tracheal”, "bronchus", "bronchial”, “rupture”, "laceration”, “injury”, "trauma”, “noniatrogenic”, "blunt” and "chest” in the title of the article. Case series discussing tracheobronchial injuries in patients with tracheostomy, lung transplant or after thoracic or cervical surgery were excluded. Many case series and review articles comprised both PiTIs and PTTBIs. 
PiTI

Epidemiology of PiTI

Considering the very high worldwide number of intubations for various reasons and the low number of publications concerning PiTI, it seems that this complication is extremely rare with no prospective studies reporting their incidence. PiTIs are probably underreported in the literature, occurring after $0.05-0.37 \%$ of orotracheal intubations [1]. The first case series was published in 1995 [2] and the largest study reported only 30 cases [3].

\section{Risk factors for PiTI}

A few patient-related risk factors have been reported such as female gender and short stature [3-5]. Context-related risk factors are mostly urgent intubations and intubations requiring rigid guides. Nonetheless, it should be kept in mind that most PiTIs occur after regular orotracheal intubations performed by experienced anaesthesiologists in the context of planned procedures [3]. Therefore, even after an easy orotracheal intubation, the diagnosis of PiTI should be considered in patients with suggestive symptoms [1, 3].

\section{Pathophysiology and identification of PiTI}

Typical PiTIs are longitudinal lacerations of the middle third of the membranous trachea. They can extend to the lower third of the trachea and exceptionally to the main bronchi. They are the direct consequence of the impact of the tip of the endotracheal tube against the membranous trachea. The depth of the lesions is variable. In extreme cases, oesophageal protrusion into the tracheal lumen during expiration can be seen. Air leaks from the trachea to the mediastinum and subcutaneous areas of the face, neck and chest.

\section{How to diagnose PiTI}

History and physical examination

The typical presentation suggestive of the diagnosis is cough and subcutaneous emphysema of the face and trunk following orotracheal intubation. Sometimes a symptom-free interval of 3 or more days after extubation can be seen [1,3]. The subcutaneous emphysema can sometimes extend to the pelvis via the retroperitoneal space. Symptoms of respiratory failure can be seen depending on the importance of the leak and the presence of an associated pneumothorax. In a patient under positive pressure ventilation, high peak and plateau pressure, tension pneumothorax or a persistent leak from a chest tube should all be suggestive of PiTI. In a mechanically ventilated patient, PiTI should be considered whenever a pneumothorax with persistent leak appears after intubation or whenever overblowing of the cuff of the endotracheal tube is required in order to stop a ventilatory leak. It should be kept in mind that this overblowing may be responsible for ischemia of the tracheal mucosa, but it probably does not induce PiTI per se, since no such cases were reported to our knowledge.

Imaging

On chest x-rays, PiTIs can be suspected using indirect signs such as pneumomediastinum extending to the neck or the thoracic wall, pneumothorax or pneumoperitoneum. Chest computed tomography (CT) is more sensitive in detecting pneumomediastinum pneumothorax or pneumoperitoneum and is the current gold standard [6]. The posterior tracheal tear is only occasionally seen.

\section{Bronchoscopy}

Endoscopic confirmation of the diagnosis of PiTI is mandatory whether it is suspected clinically or radiologically. The confirmation can be easily made in case of long and deep tears with a protruding oesophagus during cough or expiration. A careful examination during multiple respiratory cycles is sometimes necessary since the sides of the tear tend to join. The exact location of the tear should also be noted as well as its extensions to any of the main bronchi. In intubated patients, whenever a PiTI is suspected, a flexible bronchoscopy performed via the endotracheal tube does not rule out PiTI. In this context, one might wonder if a transient extubation is justified in order to explore the entire length of the trachea. Careful withdrawal of the tip of the endotracheal tube under direct bronchoscopic visualisation until the conus elasticus, should suffice. Nonetheless, in our case series, there were no PiTIs in the upper third of the trachea [3]. To our knowledge, an isolated TiBI of the upper third of the trachea has not been reported in the literature. Therefore, it is probably sufficient to withdraw slightly the endotracheal tube in order to examine the middle and lower thirds of the trachea.

\section{Management of PiTI}

Early repair by right posterolateral thoracotomy has long been the cornerstone of management of PiTI $[2,5,7]$. Good results have been claimed by some authors [8-10] as it prevents mediastinitis and scarring airway stenosis. Some criteria have also been given to carefully select patients not requiring surgery: small 
tears in haemodynamically stable patients, not requiring ventilatory assistance, and not having pneumomediastinum, mediastinitis or oesophageal lesions. In fact, whenever a transmembranous oesophageal tear is seen, mediastinitis occurs very often. Otherwise, mediastinitis as a complication of PiTI is seldom [7] reported in the literature. Secondary scarring airway stenosis can be seen in tracheobronchial lesions resulting from closed thoracic trauma but is rarely reported in PiTI. In our series, one of two patients with these complications required surgical management [3].

CARDillo et al. [11] suggested, in a prospective study of 30 patients, a morphological classification for PiTIs depending on the depth of tracheal wall involvement (I-IIIb), that could help standardise treatment but has not been validated prospectively.

Based on multiple case series [7, 12-18] and referring to our prospective study [3], more authors tend to manage PiTI conservatively even in patients with large tears (up to $9 \mathrm{~cm}$ long) (figure 1). Even in cases of transparietal rupture of the membranous trachea with an oesophageal protrusion in the tracheal lumen, often responsible for dyspnoea, noninvasive ventilation (NIV) allows both a resolution of respiratory

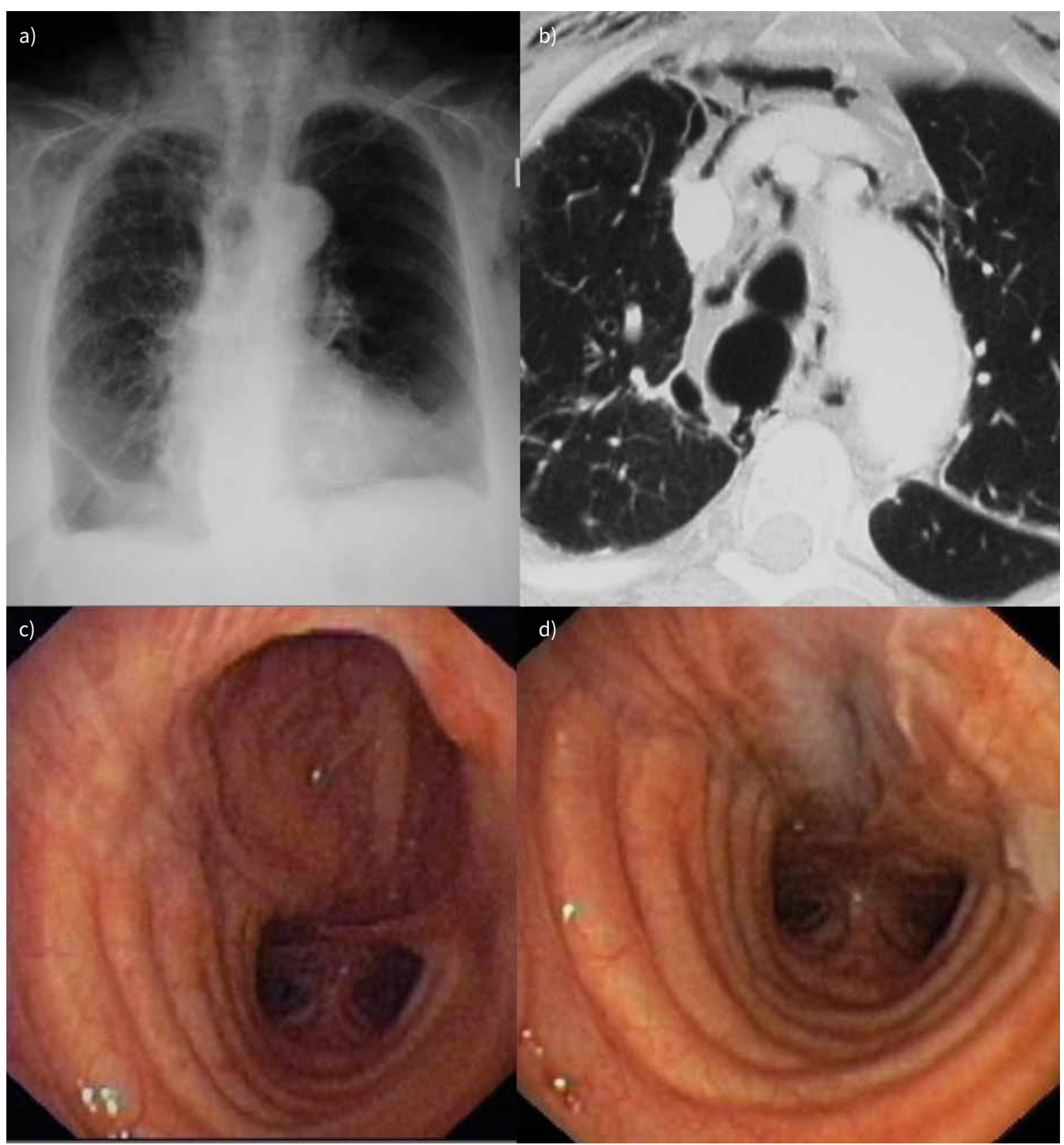

FIGURE 1 a) Chest roentgenogram: emphysema of the subcutaneous tissues of the neck and the mediastinum. b) Tomographic image of a deep tear of the membranous trachea. c) Endoscopic image of the deep tear of the membranous trachea extending over the middle and lower third of the trachea. d) In the absence of any intervention, the endoscopic examination performed after 15 days shows almost complete healing of the tear. 

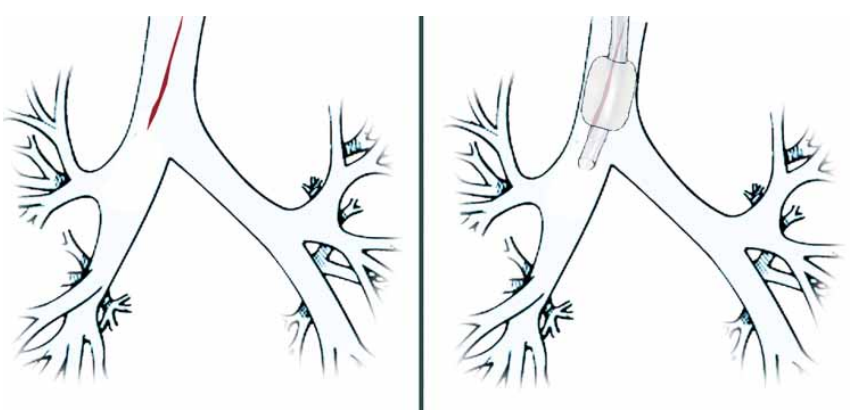

FIGURE 2 Simple bridging of a mid-third injury of the trachea.

distress and a conservative healing of the PiTI. In patients requiring mechanical ventilation for any underlying condition, healing can be improved by inserting caudally the endotracheal tube in order to bypass the tear (figure 2) or using selective bilateral endobronchial cannulation whenever the tear is distal (figure 3) [3, 12]. Based on our prospective experience and the review of the literature [3, 12, 13], we present an algorithm whereby the first decisional criterion is the need for mechanical ventilation and the second is the possibility of shunting the injury (figure 4) [19].

The management of these lesions requires a close, coordinated and rapid collaboration between anaesthesiologists, intensivists, pulmonologists and thoracic surgeons. Conservative treatment includes spontaneous ventilation, NIV or mechanical ventilation. In the latter case, the lesion is bypassed (figures 2 and 3) to avoid exposure to positive pressure. Although there are no data to support it, antibiotic prophylaxis is systematic, even if the risk of mediastinitis is low. In cases of invasive ventilation, we

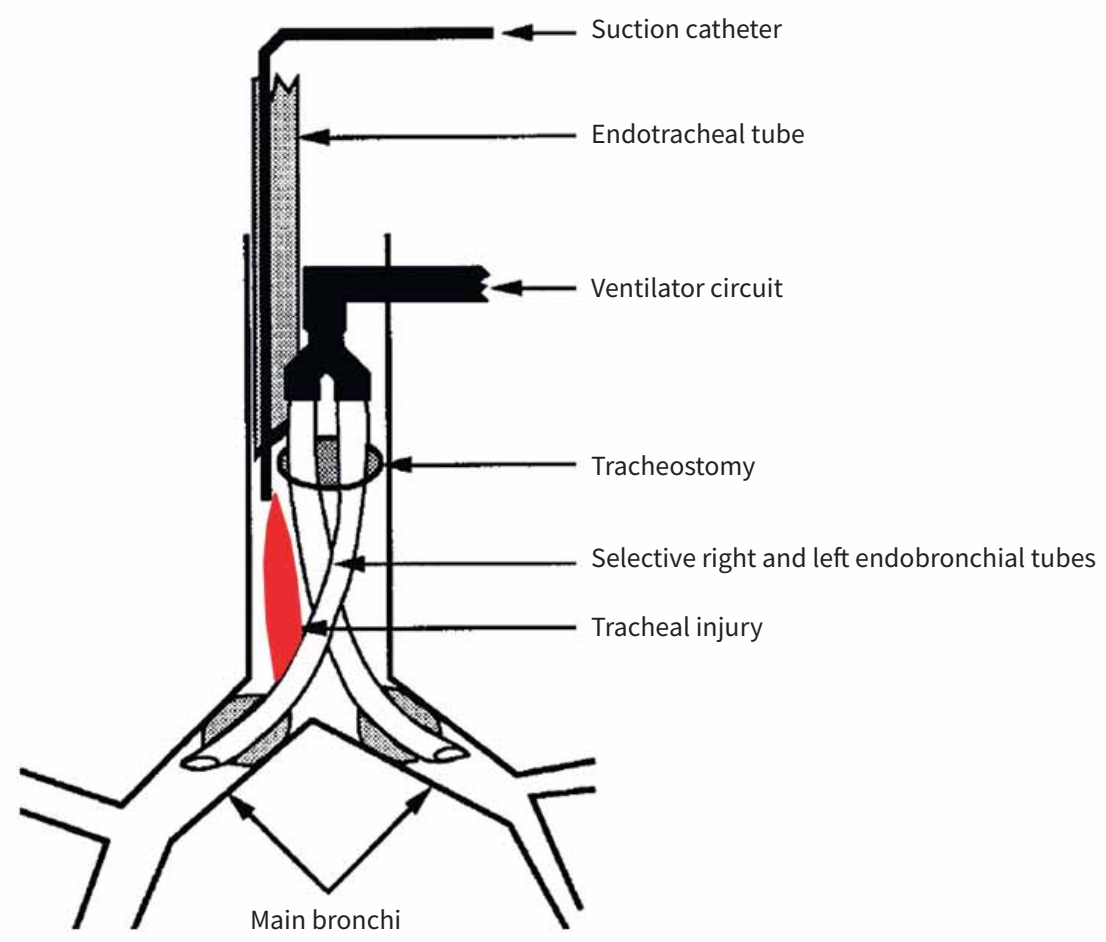

FIGURE 3 Selective right and left bronchial intubation in a patient with a peri-carinal lesion in whom surgical management was not possible due to acute respiratory distress syndrome. Reproduced from [12] with permission. 


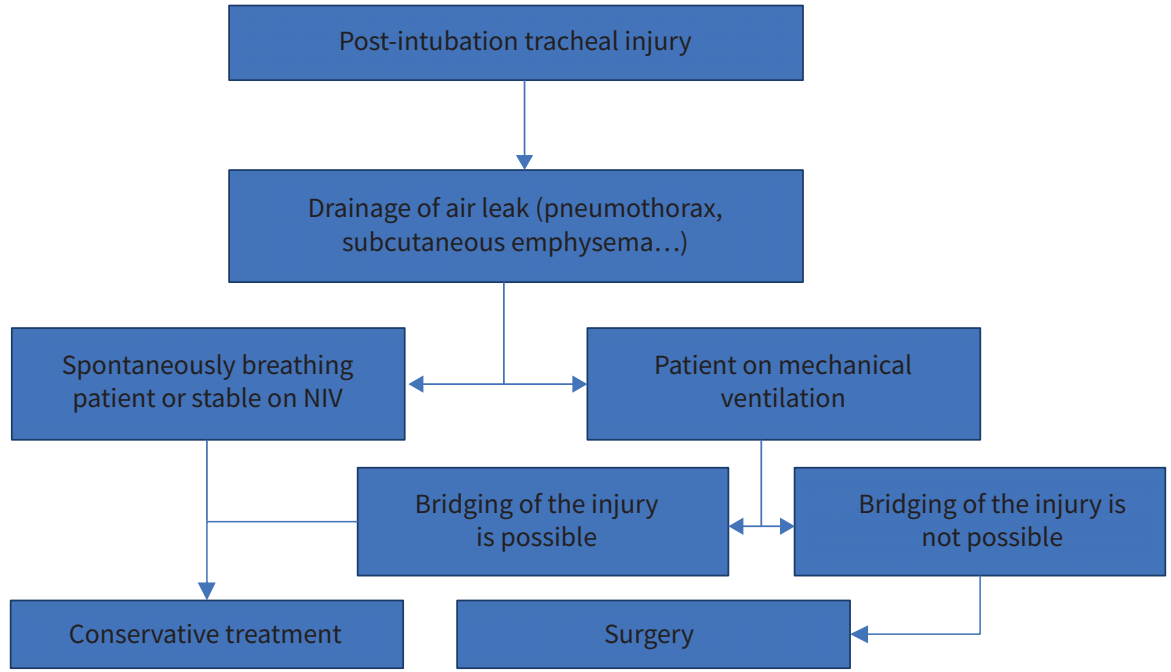

FIGURE 4 Algorithm: decision tree for post-intubation tracheal injury (adapted from references [3] and [20]). NIV: noninvasive ventilation.

suggest protective lung ventilation, good fixation of the probes using sutures to the skin in case of bilateral bronchial cannulation, maximum precautions when mobilising patients to avoid mobilisation of endobronchial tubes and regular endoscopic evaluation to allow weaning as soon as possible after the lesion heals (2-6 weeks). If the conservative option was chosen, one must be cautious of any respiratory worsening. If a surgical management is planned, the intervention must be rapidly programmed because, past the milestone of the first 48-72 h, morbidity and mortality increase significantly. This strategy has a mortality rate of around $20 \%$ [1,3]. Even if it is not yet the subject of a consensus, many teams have adopted it.

Although to our knowledge, the classification proposed by CARDILLo et al. [11] has never been validated prospectively, Grewal et al. [20] suggest in their review article a therapeutic strategy based on the classification as follows: For superficial (level I) TiBIs, the conservative option is preferred. In TiBIs involving the mucosal layer with subcutaneous or mediastinal emphysema (level II), patients are usually treated on a case-by-case basis. Patients with deep PiTIs (level III) require multidisciplinary evaluation to determine which patients need surgical or minimally invasive approaches such as airway stenting as described in some case reports [21] and based on series of short-term deployment of self-expanding metallic stents (SEMS) in tracheobronchial dehiscence following lung transplantation [22]. This proposition of management is interesting but needs to be validated prospectively.

\section{PTTB}

\section{Epidemiology of PTTBI}

The true incidence of PTTBI is not known since many patients die before arriving at the hospital. Two case series have reported an incidence of $2.1-5.3 \%$ in patients facing blunt force chest traumas that are alive on arrival [23, 24], while Bertelsen and Howitz [25] reported an incidence of $2.8 \%$ for a necropsy series. Motor vehicle accidents seem to be the most frequent mechanism of injury [26, 27]. PTTBI was often accompanied by concomitant thoracic injury such as pneumo- and haemothorax and was associated with an increase in mortality of traumatic incidents in a recent German epidemiological study [27].

What are the risk factors for PTTBI?

Most often, PTTBI results from high-energy blunt chest trauma: road traffic injuries or falls from height in young patients with a supple chest who are expecting the shock, hence against a closed glottis.

What is the pathophysiology and the observed lesions in PTTBI?

As opposed to PiTIs, PTTBIs are most often transverse or irregularly shaped disruptions involving the main carina and often extending into the main bronchi, i.e. they may be between the cartilage rings or in 
spiral shape going from the membranous trachea down to one of the main bronchi. In most cases the tear is in a $2.5 \mathrm{~cm}$ perimeter around the carina.

Three mechanisms can intervene alone or in combination and account for the large predominance of lesions at the level of the posterior wall of the lower third of the trachea and the main bronchi or at the level of the carinal division [26].

1. High-energy anteroposterior compression resulting in a marked decrease in the anteroposterior diameter of the chest and therefore a widening of the thorax and a lateral stretching of the main bronchi across the carina. Tracheobronchial rupture can occur whenever the exerted force exceeds the elasticity of the tracheobronchial tissue.

2. Sudden deceleration during which the maximum shearing force is exerted at the two fixed points, in this case, the cricoid and the carina. The former is attached to the thyroid cartilage while the bronchopericardial membrane binds the latter to the pericardium. Rupture occurs if the shear force is strong enough to tear the tissue.

3. Crushing of the trachea and main bronchi between the spine and the sternum. The air cannot escape when the glottis is closed (in a subject who anticipates the trauma). Consequently, there is a sudden and marked increase in the intra-luminal pressure, the largest increase being that of wall tension in the main respiratory tract with rupture occurring in the most fragile areas (posterior membranous or at the angle between membranous and tracheal rings). In the elderly, such trauma will lead to multi-level rib fractures with costal flap, thus avoiding sufficient intra-luminal pressure to create PTTBI. Although it has not been studied in the literature, in practice, it is rare to observe PTTBI in patients with multi-stage rib fractures.

\section{How to diagnose PTTBI}

The approach is not fundamentally different from the diagnostic approach for PiTI and combines clinical, radiological and endoscopic signs.
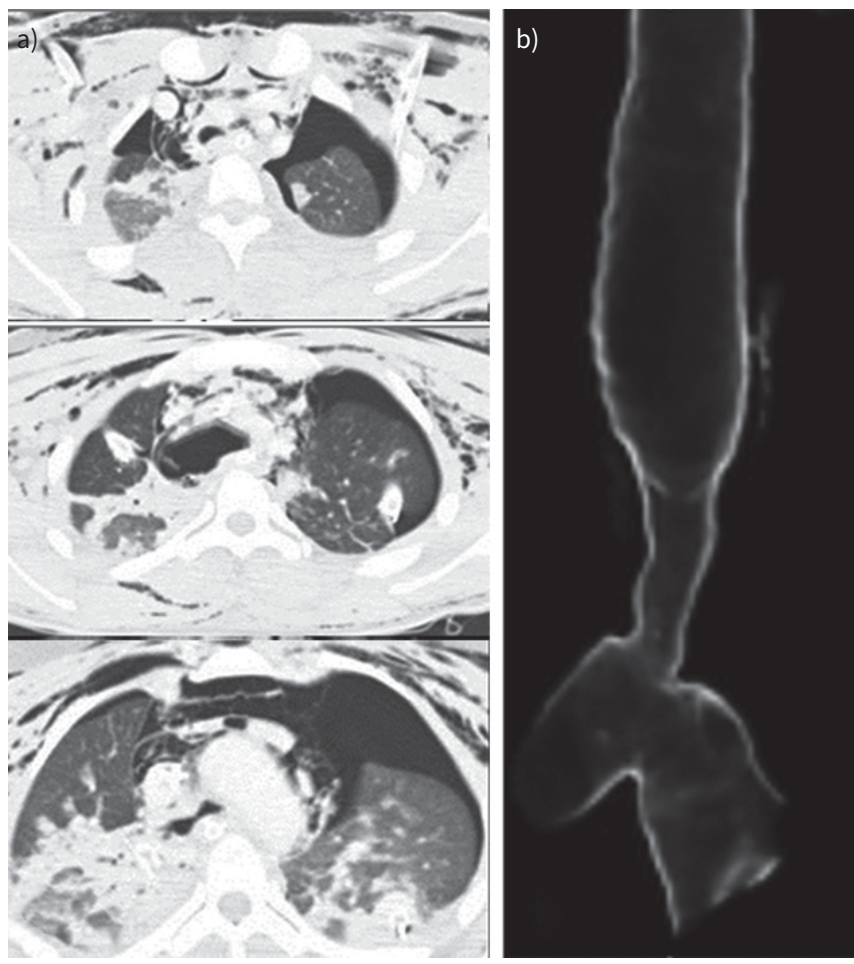

FIGURE 5 a) Young female patient in a state of shock, coma and acute respiratory failure following a car accident. High-energy blunt chest trauma present. Total body scan showed liver contusion, cerebral oedema and pulmonary contusion. b) 3 months later, while the patient had recovered, she presented with laboured breathing associated with severe stenosis of the lower third of the trachea. Retrospectively, the body scan showed complete rupture of the lower third of the trachea that had gone unnoticed. 
Clinical

The clinical signs are similar to those of PiTIs except they are more severe (major subcutaneous emphysema, respiratory failure) and occur more rapidly. Haemoptysis occurs in 25\% of cases, related to bleeding from the mucosa. Abundant haemoptysis could suggest an associated vascular lesion. The diagnosis can be difficult, either because subcutaneous emphysema and pneumothorax are considered complications of possibly associated rib fractures or because traumatic lesions or failures associated with PTTBI dominate the clinical picture (figure 5). It is essential to bear in mind, for a mechanically ventilated polytraumatised patient with a chest tube, that whenever an air leak is ongoing and pneumothorax persists, PTTBI should be suspected and sought.

\section{Imaging}

In polytrauma patients, subcutaneous emphysema and pneumothorax are not always complications of costal fractures. In the event of pneumothorax, two signs should strongly suggest PTTBI: the "fallen lung sign” due to a complete rupture of a main bronchus and the persistence of pneumothorax despite drainage. In addition, one should always consider, in a patient with polytrauma, a tear in the tracheal membranous membrane whenever an overinflated cuff of the endotracheal tube is seen on chest imaging; that is whenever nurses need to overinflate the cuff in order to obtain an acceptable pressure.

The CT scan often shows non-specific signs in a blunt chest trauma such as pneumomediastinum or pneumothorax. Sometimes it shows the tracheal or bronchial tear. Special attention should be given to detecting lesions occurring in high-energy blunt trauma patients, including lesions of the large vessels. Multiplanar reconstruction and 3D reconstruction of the airway can be valuable to establish a definitive diagnosis and can help the surgeon decide on the optimal surgical approach $[28,29]$.

\section{Bronchoscopy}

Bronchoscopy is the key examination that allows the diagnosis and assessment of tracheobronchial tears. The examination should be performed by an experienced bronchoscopist as the aspects are misleading. A clear tracheal or bronchial tear is exceptional. Therefore, it may not be noticed especially if the adventitia of the trachea contains the fracture. Most often, the endoscopic diagnosis is indirect: the bronchial axis is

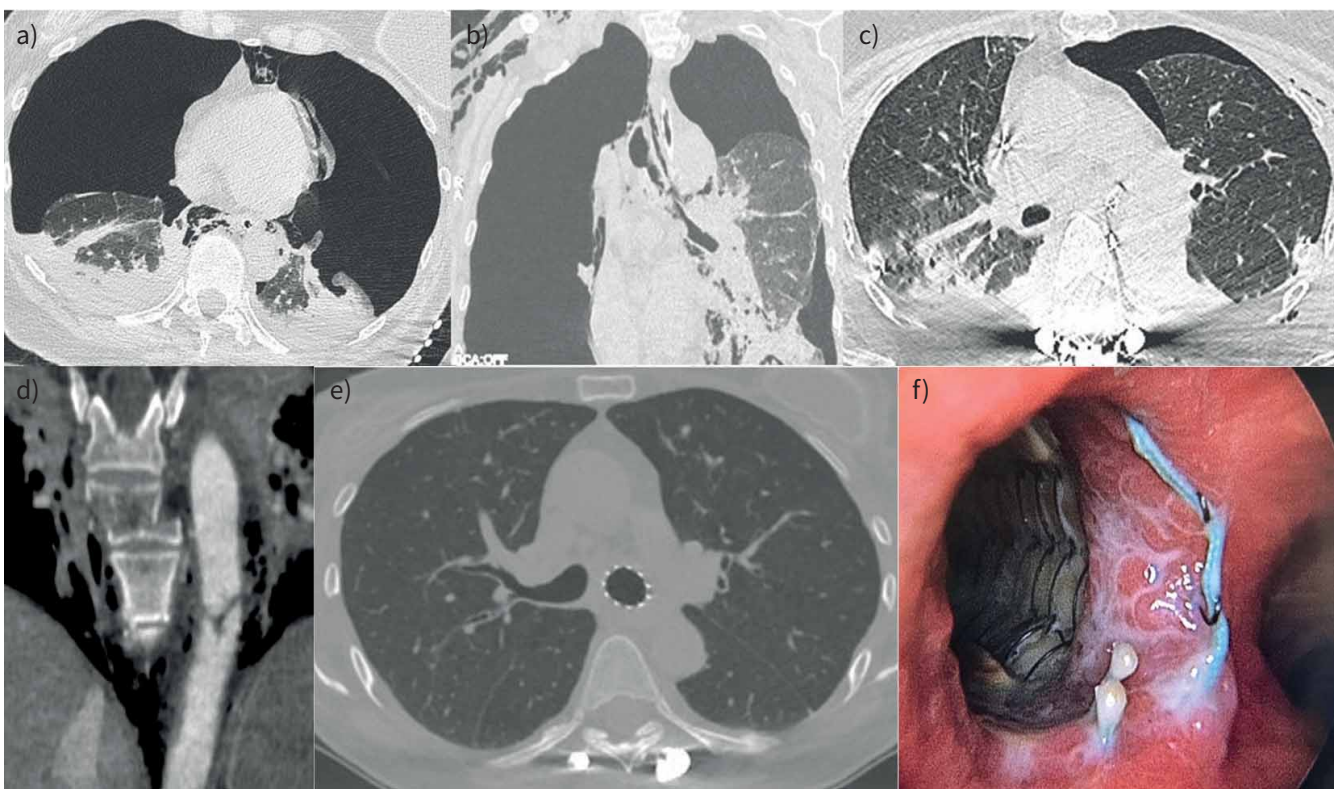

FIGURE 6 a) and b) 57-year-old female in acute respiratory failure after a fall. c) The patient was intubated, and bilateral chest tubes inserted. d) A displaced fracture of the 10th dorsal vertebra, an aortic dissection and a complex fracture of the left main bronchus just under the aortic arch are seen on the total body scan. Endoscopically, a tight stenosis of the left main bronchus is seen. On day five, and due to the contra-indication to immediate surgery, endoscopic recanalisation of the left bronchial tree using a guidewire allowed the deployment of a self-expanding metallic stent of the left main bronchus (e, f). The stent was removed 3 months later with satisfactory healing. 
deviated, obstructed by a bleeding mucosa or clots, thus a differential diagnosis with a tumoral stenosis (figure 6).

\section{How is PTTBI treated?}

Unlike PiTI, there is little room for conservative management for two reasons: 1) ventilatory leaks can complicate mechanical ventilation; and 2) There is a risk of stricture and abnormal healing (figure 5). This has been reported in multiple case series [30-33]. The first step of treatment should be securing the airway. Flexible bronchoscopy allows the repositioning of the endotracheal tube in order to bypass the injury. In a patient with a damaged main bronchus, with respiratory failure requiring intubation, endoscopically guided selective intubation in the contralateral bronchus is required. In the event of a tracheal lesion, the endotracheal tube should be guided into the left main bronchus because of the usual right posterolateral approach of the thoracotomy in this case. Switching the regular endotracheal tube for a Carlens double-lumen tube in an already damaged trachea not advisable and may worsen the lesions due to its larger size, its more delicate introduction and the risk of losing the airway in the process. In spontaneously breathing patients, in the absence of oesophageal injury and respiratory or circulatory failure, transverse lesions extending to less than the third of the circumference of the airway or small longitudinal tears $(<2 \mathrm{~cm})$ the air leakage of which is well controlled by drainage can be treated conservatively [34].

Surgery should probably not be deferred in this context. One retrospective study [27] showed higher mortality in patients undergoing surgery within the first $24 \mathrm{~h}$ after the impact. Another retrospective study [33] showed a lower mortality whenever surgery was performed during the first $2 \mathrm{~h}$ after the impact. In a context of retrospective studies, no firm conclusions can be drawn. Associated injuries, in both studies, were associated with a higher mortality rate.

Once the surgical decision has been made, the approach depends on the location of the lesion. When the tear concerns the trachea, right main bronchus or proximal left main bronchus, a right posterolateral thoracotomy is performed for optimal exposure to the lesion. If the lesion is distal in the left main bronchus ( $>2 \mathrm{~cm}$ from the carina), a left posterolateral thoracotomy is preferred [24, 25].

In "desperate" cases in which the treatment of associated traumatic lesions dominated the initial therapeutic management, interventional endoscopy, as an alternative or bridge to surgery, allows for restitution of alignment of a fractured and distorted bronchus using covered SEMS (figure 6). They should be carefully chosen by avoiding over- or under-sizing which could lead to delayed healing or migration respectively. In case they are used, stent maintenance using humidifying nebulisation or mucolytics to avoid clogging should be prescribed. A regular bronchoscopic evaluation is advisable as long-term complications (granulation tissue, colonisation, perforation, migration) are dreadful. Some authors have also reported successful stenting of delayed fibrotic airway stenosis after conservative treatment [35].

\section{Conclusion}

PiTIs are exceptional complications of intubation. Their early endoscopic diagnosis allows conservative treatment, especially in patients with spontaneous ventilation or when the lesion can be bridged by the endotracheal tube.

PTTBIs are rare but potentially serious complications of closed chest trauma. The clinical presentation can be nonspecific and clinicians should have a high index of suspicion in this type of trauma. Persistent air leakage after well-conducted pleural drainage justifies a thorough endoscopic assessment. Early diagnosis by bronchoscopy and selective intubation allows for early management of these lesions and their good functional recovery.

Close, coordinated and rapid collaboration between anaesthesiologists, intensivists, pulmonologists and thoracic surgeons is a key factor to success in the management of these lesions.

Provenance: submitted article, peer reviewed.

Conflict of interest: None declared.

\section{References}

1 Miñambres E, Burón J, Ballesteros MA, et al. Tracheal rupture after endotracheal intubation: a literature systematic review. Eur J Cardiothorac Surg 2009; 35: 1056-1062. 
2 Marty-Ané $\mathrm{CH}$, Picard E, Jonquet O, et al. Membranous tracheal rupture after endotracheal intubation. Ann Thorac Surg 1995; 60: 1367-1371.

3 Conti M, Fournier C, Hysi I, et al. Conservative management of postintubation tracheal membrane ruptures. Intensive Care Med 2010; 36: 1622-1623.

4 Hofmann HS, Rettig G, Radke J, et al. latrogenic ruptures of the tracheobronchial tree. Eur J Cardiothorac Surg 2002; 21: 649-652.

5 Massard G, Rougé C, Dabbagh A, et al. Tracheobronchial lacerations after intubation and tracheostomy. Ann Thorac Surg 1996; 61: 1483-1487.

6 Caceres M, Ali SZ, Braud R, et al. Spontaneous pneumomediastinum: a comparative study and review of the literature. Ann Thorac Surg 2008; 86: 962-966.

7 Kaloud H, Smolle-Juettner FM, Prause G, et al. latrogenic ruptures of the tracheobronchial tree. Chest 1997; 112: 774-778.

8 Mussi A, Ambrogi MC, Menconi G, et al. Surgical approaches to membranous tracheal wall lacerations. J Thorac Cardiovasc Surg 2000; 120: 115-118.

9 Sippel M, Putensen C, Hirner A, et al. Tracheal rupture after endotracheal intubation: experience with management in 13 cases. Thorac Cardiovasc Surg 2006; 54: 51-56.

10 Borasio P, Ardissone F, Chiampo G. Post-intubation tracheal rupture. A report on ten cases. Eur J Cardiothorac Surg 1997; 12: 98-100.

11 Cardillo G, Carbone L, Carleo F, et al. Tracheal lacerations after endotracheal intubation: a proposed morphological classification to guide non-surgical treatment. Eur J Cardiothorac Surg 2010; 37: 581-587.

12 Marquette $\mathrm{CH}$, Bocquillon $\mathrm{N}$, Roumilhac $\mathrm{D}$, et al. Conservative treatment of tracheal rupture. J Thorac Cardiovasc Surg 1999; 117: 399-401.

13 Conti M, Pougeoise M, Wurtz A, et al. Management of postintubation tracheobronchial ruptures. Chest 2006; 130: 412-418.

14 Gómez-Caro A, Ausín P, Moradiellos FJ, et al. Role of conservative medical management of tracheobronchial injuries. J Trauma 2006; 61: 1426-1434.

15 Carbognani P, Bobbio A, Cattelani L, et al. Management of postintubation membranous tracheal rupture. Ann Thorac Surg 2004; 77: 406-409.

16 Singh S, Gurney S. Management of post-intubation tracheal membrane ruptures: a practical approach. Indian J Crit Care Med 2013; 17: 99-103.

17 d'Odemont JP, Pringot J, Goncette L, et al. Spontaneous favorable outcome of tracheal laceration. Chest 1991; 99: 1290-1292.

18 Jougon J, Ballester M, Choukroun E, et al. Conservative treatment for postintubation tracheobronchial rupture. Ann Thorac Surg 2000; 69: 216-220.

19 Wurtz A, Benhamed L, Hysi I, et al. Endoscopic or conservative management of postintubation tracheal membrane laceration. Ann Thorac Surg 2011; 91: 1654-1655.

20 Grewal HS, Dangayach NS, Ahmad U, et al. Treatment of tracheobronchial injuries: a contemporary review. Chest 2019; 155: 595-604.

21 Marchese R, Mercadante S, Paglino G, et al. Tracheal stent to repair tracheal laceration after a double-lumen intubation. Ann Thorac Surg 2012; 94: 1001-1003.

22 Mughal MM, Gildea TR, Murthy S, et al. Short-term deployment of self-expanding metallic stents facilitates healing of bronchial dehiscence. Am J Respir Crit Care Med 2005; 172: 768-771.

23 Iwasaki M, Kaga K, Ogawa J, et al. Bronchoscopy findings and early treatment of patients with blunt tracheo-bronchial trauma. J Cardiovasc Surg 1994; 35: 269-271.

24 de la Rocha AG, Kayler D. Traumatic rupture of the tracheobronchial tree. Can J Surg J Can Chir 1985; 28 : 68-71.

25 Bertelsen S, Howitz P. Injuries of the trachea and bronchi. Thorax 1972; 27: 188-194.

26 Kiser AC, O'Brien SM, Detterbeck FC. Blunt tracheobronchial injuries: treatment and outcomes. Ann Thorac Surg 2001; 71: 2059-2065.

27 Schibilsky D, Driessen A, White WJ, et al. Traumatic tracheobronchial injuries: incidence and outcome of 136,389 patients derived from the DGU trauma register. Sci Rep 2020; 10: 20555.

28 Scaglione M, Romano S, Pinto A, et al. Acute tracheobronchial injuries: impact of imaging on diagnosis and management implications. Eur J Radiol 2006; 59: 336-343.

29 Faure A, Floccard B, Pilleul F, et al. Multiplanar reconstruction: a new method for the diagnosis of tracheobronchial rupture. Intensive Care Med 2007; 33: 2173-2178.

30 Richardson JD. Outcome of tracheobronchial injuries: a long-term perspective. J Trauma 2004; 56: 30-36.

31 Huh J, Milliken JC, Chen JC. Management of tracheobronchial injuries following blunt and penetrating trauma. Am Surg 1997; 63: 896-899.

32 Zhao Y, Jiao J, Shan Z, et al. Effective management of main bronchial rupture in patients with chest trauma. Thorac Cardiovasc Surg 2007; 55: 447-449. 
33 Balci AE, Eren N, Eren S, et al. Surgical treatment of post-traumatic tracheobronchial injuries: 14-year experience. Eur J Cardiothorac Surg 2002; 22: 984-989.

34 Carretta A, Melloni G, Bandiera A, et al. Conservative and surgical treatment of acute posttraumatic tracheobronchial injuries. World J Surg 2011; 35: 2568-2574.

35 Roh JY, Kim I, Eom JS, et al. Successful stenting for bronchial stenosis resulting from blunt airway trauma. Intern Med 2018; 57: 3277-3280. 